\title{
A neuronal retuning hypothesis of sentence-specificity in Broca's area
}

\author{
William G. Matchin ${ }^{1}$
}

Published online: 22 September 2017

(C) Psychonomic Society, Inc. 2017

\begin{abstract}
It is clear that the left inferior frontal gyrus (LIFG) contributes in some fashion to sentence processing. While neuroimaging and neuropsychological evidence support a domain-general working memory function, recent neuroimaging data show that particular subregions of the LIFG, particularly the pars triangularis (pTri), show selective activation for sentences relative to verbal working memory and cognitive control tasks. These data suggest a language-specific function rather than a domain-general one. To resolve this apparent conflict, I propose separating claims of domain-generality and specificity independently for computations and representations - a given brain region may respond to a specific representation while performing a general computation over that representation, one shared with other systems. I hypothesize that the pTri underlies a language-specific working memory system, comprised of general memory retrieval/attention operations specialized for syntactic representations. There is a parallelism of top-down retrieval function among the phonological and semantic levels, localized to the pars opercularis and pars orbitalis, respectively. I further explore the idea of how such a system emerges in the human brain through the framework of neuronal retuning: the "borrowing" of domaingeneral mechanisms for language, either in evolution or development. The empirical data appear to tentatively support a developmental account of language-specificity in the pTri, possibly through connections to the posterior superior temporal sulcus (pSTS), a region that is both anatomically distinct for humans and functionally essential for language. Evidence of representational response specificity obtained from
\end{abstract}

William G. Matchin

wmatchin@ucsd.edu

1 Department of Linguistics, UC San Diego, La Jolla, CA 92093, USA neuroimaging studies is useful in understanding how cognition is implemented in the brain. However, understanding the shared computations across domains and neural systems is necessary for a fuller understanding of this problem, providing potential answers to questions of how specialized systems, such as language, are implemented in the brain.

Keywords Broca's area, Working memory, Syntax, fMRI

\section{Introduction}

Whether language is governed by domain-general or domainspecific properties, whether it is innate or emergent - these have been central questions of investigation for the last several decades (Chomsky, 1959; Pinker, 1995; Saffran et al., 1996; Tomasello, 2009). A scientifically rigorous approach to these questions is not to ask either/or but rather to determine what language is; this includes identifying which parts can be attributed to more cognitively general capacities, which parts are language-specific (if any), and how they interact (Hauser et al., 2002). I pursue this approach with respect to the neurobiology of sentence processing, concerning the function of the brain region most closely associated with language: Broca's area. Broca's area is commonly defined as the posterior two thirds of the left inferior frontal gyrus (LIFG) (Fig. 1), comprised of the pars opercularis (pOper) and the pars triangularis (pTri); many authors also include the most anterior and inferior portion, the pars orbitalis (pOrb) (Tremblay \& Dick, 2016). These areas roughly correspond to Brodmann's areas 44, 45, and 47 (Amunts et al., 2010); however, because direct cytoarchitectonic information is not available in neuroimaging and neuropsychological studies, I will restrict discussion to the readily identifiable anatomical regions. 


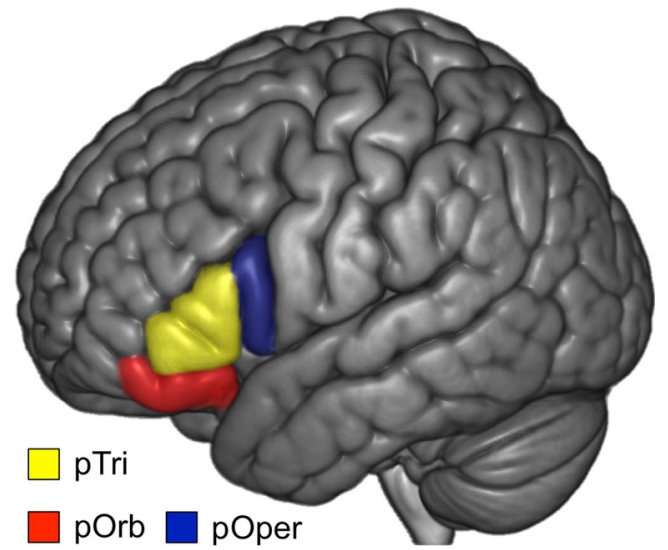

Fig. 1 The left inferior frontal gyrus (LIFG), divided into standard anatomical subregions. pOper $=$ pars opercularis, $\mathrm{pTri}=$ pars triangularis, $\mathrm{pOrb}=$ pars orbitalis

This paper chiefly concerns the $\mathrm{p}$ Tri. The $\mathrm{p}$ Tri presents an interesting case for the approach outlined above given that this region exhibits both domain-general (Rogalsky \& Hickok, 2011) and language-specific (Fedorenko et al., 2012) properties. This forces us to develop a hypothesis of this region's function that eschews simple classification into one or the other category. The idea that I pursue is that a brain region may be both domain-general and domain-specific at the same time; it may take a very specific representational input, but perform a general computation over that input. Essentially, we must break down the notions "domain-specific" and "domaingeneral" separately with respect to representations and computations. I propose that a general computational operation of the LIFG is top-down memory retrieval for representations stored in long-term memory, which is executed via wellestablished white matter tracks connecting the frontal and temporal lobes (Friederici et al., 2006; Saur et al., 2010). The three subregions of the LIFG are differentiated by the nature of the information that is retrieved; pOper operates over phonological representations, pTri operates over syntactic representations, and pOrb operates over semantic representations. Phonological and semantic retrieval are domain-general with respect to language in the sense that they are not specific to language, likely operating in other organisms and in nonlinguistic human cognition. However, top-down syntactic memory access operations (in the sense of Lewis \& Vasishth, 2005) are language-specific, given the use of syntactic features that are only found in language. This analysis raises further questions concerning the origins of linguistic specificity in the pTri. I present a framework of neuronal retuning, which suggests that this specificity arises as a result of either phylogenetic repurposing (exaptation) of top-down memory access operations applied to a novel syntactic domain (Jacob, 1977; Gould \& Vrba, 1982), or ontogenetic repurposing (neuronal recycling), that is, the emergence of domain-specificity of a brain region during development
(Dehaene \& Cohen, 2007). Both of these possibilities provide specific insights into how domain-general computations and domain-specific representations contribute to human language.

\section{Language-specific and domain-general accounts of Broca's area}

Broca's area has a long history in the discussion of the neural infrastructure of language, not the least of which is the prominent association of this region with core linguistic computations, which most authors term syntax. The most fundamental and universal property of human language is its hierarchical structure. Linguistic theory often posits a fundamental distinction between the lexicon (the stored elements of language) and the combinatorial system, or syntax (Chomsky, 1965; Jackendoff, 2002; see Sprouse \& Hornstein, 2016 for an accessible introduction). ${ }^{1}$ The lexicon is the repository of words; more technically, lexical elements. Each element includes various features that identify its meaning (semantic features), how it sounds and is produced (phonetic features), and the way it combines with others (syntactic features). The syntax is the machinery that generates potentially infinitely long sentences by combining lexical elements into hierarchical phrases and sentences. This syntactic property has been hypothesized to be a uniquely human and language-specific mechanism (Bolhuis et al., 2014), and understanding its neural implementation is of great interest if we are to understand how the human brain gives rise to human language.

Some authors (Friederici, 2011; Grodzinsky \& Santi, 2008; Hagoort, 2005) have argued that syntactic operations are implemented in Broca's area on the basis of neuropsychological evidence implicating it in sentence comprehension deficits (Caramazza \& Zurif, 1976; Grodzinsky, 2000) and functional magnetic resonance imaging (fMRI) or positron emission tomography (PET) studies finding activation in this region to syntactically complex sentences and syntactic violations (Bornkessel et al., 2005; Embick et al., 2000; Stromswold et al., 1996). In addition, fMRI (Moro et al., 2001; Pallier et al., 2011) and electrocorticography (ECoG) data (Sahin et al., 2009; Nelson et al., 2017) have revealed that the pTri, among other regions mostly in the temporal lobe, shows activity that is tightly correlated with syntactic structure and grammatical morphology, and in some studies shows

\footnotetext{
${ }^{1}$ Some authors disagree about the relative richness of the lexicon and the syntax, with some recent theories moving towards minimizing the syntax in favor of a rich lexicon (e.g., Chomsky, 1995; Frank, 2002; Jackendoff, 2002; Goldberg, 1995). However, for the purposes of this discussion, I will assume the basic dichotomy between the lexicon and syntax that most authors assume at some level. Please see Lewis \& Vasishth (2005), with respect to the memory architecture assumed here, for discussion of the workload tradeoff between the lexicon and the syntax.
} 
increased activity for very simple structured phrases and sentences compared with word lists (Zaccarella et al., 2017; Matchin et al., 2017). However, other researchers (Bornkessel-Schlesewsky \& Schlesewsky, 2013; Kaan \& Swaab, 2002; Novick et al., 2005; Rogalsky \& Hickok, 2011) point out the strong evidence implicating Broca's area in functions not specific to sentences, such as phonological working memory and cognitive control (Braver et al., 1997; Rogalsky et al., 2008; Thompson-Schill et al., 1997), maintaining that activations to sentences and comprehension deficits resulting from damage to this region are attributable to these more general mechanisms rather than syntactic operations, with syntax being implemented elsewhere. Evidence in favor of this assertion is that the activation profile during sentence processing in Broca's area is heavily task dependent and does not reliably activate for contrasts of structure, as appears to be the case in the anterior temporal lobe (ATL) (Mazoyer et al., 1993; Stowe et al., 1998; Rogalsky \& Hickok, 2009; Matchin et al., 2014; Matchin et al., 2017).

A powerful argument against a role for Broca's area in core syntactic operations is that damage to Broca's area is not associated with notable language or sentence comprehension deficits (Mohr et al., 1978; Dronkers et al., 2004; Thothathiri et al., 2012; cf Davis et al., 2008). Patients with lesions to Broca's area that do not encroach on the temporal lobe also are mostly capable of making subtle acceptability judgments or decisions about the well-formedness of sentences (Linebarger et al., 1983; Wilson \& Saygin, 2004). Acceptability judgments are the core data underlying syntactic theories; making these judgments likely requires intact syntactic abilities. By contrast, sentence comprehension and acceptability judgment deficits are strongly associated with posterior temporal lobe (PTL) and angular gyrus lesions (Dronkers et al., 2004; Thothathiri et al., 2012; Mesulam et al., 2015; Wilson \& Saygin, 2004; Pillay et al., 2017). Any account of the function of Broca's area in language must account for the fact that patients with damage restricted to this region have such intact abilities.

Amid this debate, several recent studies (Fedorenko et al., 2012; Bedny et al., 2011; Rogalsky et al., 2015) have reported a high degree of sentence-specific activations in Broca's area, particularly in the pTri. For example, Fedorenko et al. (2012) found subregions that activate to sentences more than lists of nonwords but do not respond to nonsentence tasks, including phonological working memory and cognitive control (Fig. 2). Consistent with this, an fMRI study by Rogalsky et al. (2008) showed that a classic contrast of noncanonical sentences > canonical sentences known to induce processing difficulties (object-relatives vs. subject relatives) revealed activity in both the pTri and pOper; a secondary task of subvocal articulatory

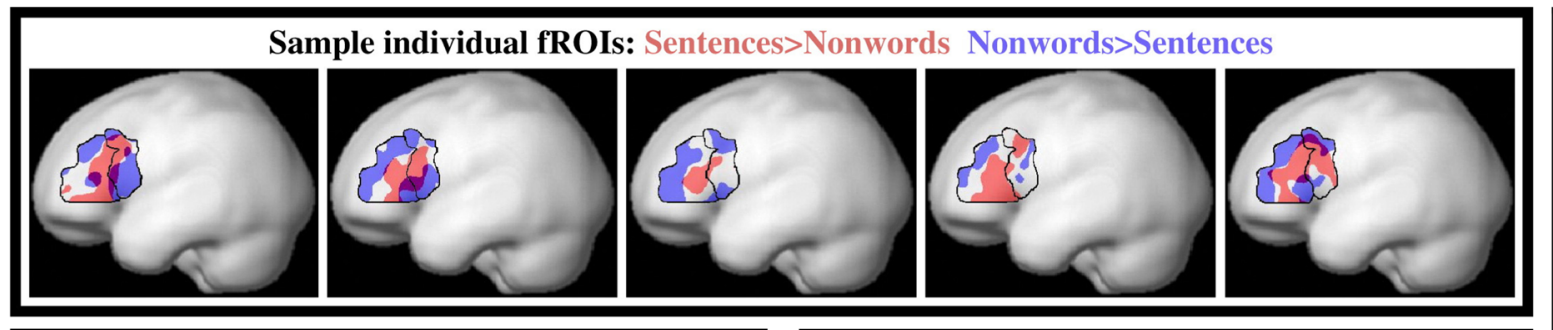

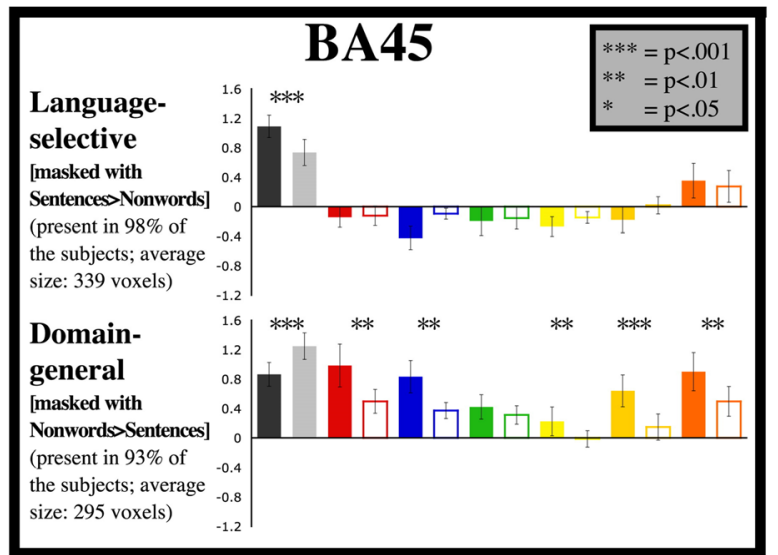

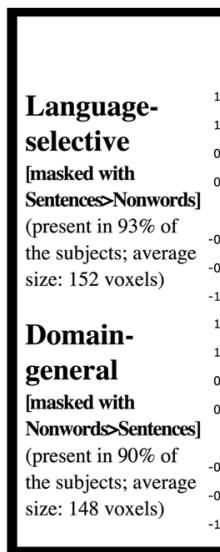

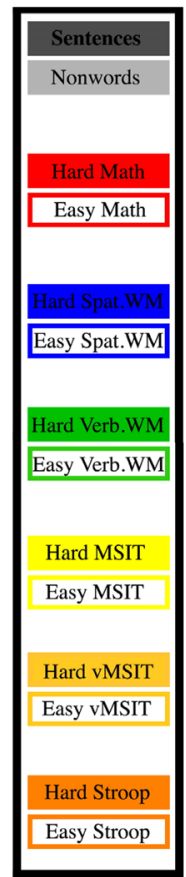

Fig. 2 Language-selective and domain-general subregions of Broca's area (adapted and modified from (Fedorenko et al., 2012) under the Creative Commons Attribution License). RIGHT: the various tasks presented to subjects while in the scanner. TOP: ROIs identified by the language localizer runs. Red areas indicate regions more active for sentences than lists of nonwords (language-selective), and blue indicates regions more active for lists of nonwords compared to sentences (domain- general). Outlines of BA44 and BA45 are shown in black lines. BOTTOM: activation for each task within the obtained ROIs in individual subjects. Domain-general tasks activate langauge-selective ROIs minimally or not at all, while strongly activating the domain-general ROIs. Note that BA45 roughly corresponds to the pTri, and most activations appear to be centered on the pTri rather than pOper 
rehearsal eliminated activity in the pOper but not in the pTri. Thus, while activity for sentence processing in the pOper can be reasonably attributed to subvocal articulation associated with verbal working memory, activations in the pTri cannot. These results challenge working-memory and cognitive control hypotheses about the function of Broca's area in sentence processing, because these hypotheses expect brain areas that activate for sentence processing also to activate for nonsentence tasks, such as verbal working memory. The linguistic specificity of the pTri is similar to the fusiform face area (FFA), which has a neuroimaging response profile selective for faces compared with other complex visual stimuli (Kanwisher et al., 1997; McCarthy et al., 1997). The essential and selective role of the FFA in face processing suggested by its face-specific response profile is supported by studies that have shown that lesions to the FFA produce prosopagnosia, or face blindness (Barton, 2008). The parallelism in neuroimaging specificity between the FFA and Broca's area thus suggests a parallelism of function (Fedorenko \& ThompsonSchill, 2014), and some authors therefore suggest that these regions underlie core linguistic computations, arguing that domain-general mechanisms cannot account for these language-specific patterns (Fedorenko \& Thompson-Schill, 2014)

It is clear that claims of a hard parallelism of function between the FFA and pTri are untenable, because lesions selective to the LIFG do not typically produce profound language deficits in the way that lesions to the temporal lobe do. In addition, a core linguistic function (e.g., syntactic operations) does not adequately explain the pattern of sentence-related activity in the $\mathrm{pTri}$ across neuroimaging studies. The challenge is to develop a hypothesis of this region's function that accounts for all the data combined. In this context, let us step back and reflect on what a neuroimaging response profile for domain-specific representations might indicate with respect to the function of a given brain area. There are at least two logical possibilities: (i) this brain area performs a function completely independent of the representations and computations of other brain regions, or (ii) this brain area is specialized for a particular representational domain, yet performs a general computation shared with other neural systems. In the following sections, I pursue the latter option: pTri underlies a syntactic memory retrieval system. This system is domain-general with respect to its computational properties, namely top-down memory retrieval operations, and domainspecific with respect to the information that is retrieved, namely syntactic representations, as posited by McElree et al. (2003), Lewis et al. (2006), and Caplan \& Waters (2013). This proposal agrees with previous proposals of neuroimaging researchers who posited that the function of the pTri is syntactic working memory (Fiebach et al., 2005; Rogalsky et al., 2015). This could be seen as an extension of Baddeley's phonological loop subsystem to a "syntactic loop," although the memory retrieval architecture outlined by the authors above is distinct from the maintenance architecture of the Baddeley model (Baddeley \& Hitch, 1974; Baddeley, 2003). In the following sections, I motivate the existence of language-specific working memory resources, as well as how such a specialized system emerges in the human brain.

\section{Syntactic working memory}

Two central claims of this paper are that there exists a working memory system specific to sentence processing and that this system is localized roughly to the pTri. It is uncontroversial that the $\mathrm{pTri}$, a region of the prefrontal cortex of humans, might have a function related to working memory. Working memory is a capacity present in nonhuman, nonlinguistic animals, with the prefrontal cortex playing a central role in this capacity. Persuasive evidence for this is sustained neural activity in single neurons in prefrontal regions over a delay period in which a stimulus must be maintained and impairments on working memory tasks when this region is lesioned (Goldman-Rakic, 1995; Murphy et al., 1996; Miller et al., 1996; Zahrt et al., 1997). Evidence for the existence of working memory resources in the prefrontal cortex of humans, particularly in the vicinity of Broca's area, also is well-established (Braver et al., 1997; Thompson-Schill et al., 1997; Curtis \& D'Esposito, 2003; Badre \& Wagner, 2007; Glaser et al., 2013). These neuroimaging experiments also show sustained activity in the prefrontal cortex over delay periods (Curtis \& D'Esposito, 2003), for comparisons of stimuli incurring differing working memory demands (Braver et al., 1997), and specifically for memory retrieval interference effects during sentence processing (Glaser et al., 2013).

While language is a human-specific trait and involves unique structural properties (Hauser et al., 2002; Bolhuis et al., 2014), there is strong evidence that aspects of successful sentence processing rely on working memory resources. Observations have long been made about particular structural configurations that pose problems for comprehension, such as multiple self-embedding (Miller \& Chomsky, 1963) (e.g., the rat that the cat that the dog chased ate died is difficult to understand, whereas the dog chased the cat that ate the rat that died poses much less problem). These difficulties appear to be due to the high working memory demands these configurations create (Gibson, 1998; Lewis et al., 2006). In addition, it is likely that simple sentence structures engage working memory under conditions of time constraints, noisy environments, or when speakers create false starts and stops of sentences.

Less obvious is whether there are working memory resources that are dedicated to sentence processing (see Just \& Carpenter, 1992; Caplan \& Waters, 1999, 2013; Fedorenko et al., 2007 for discussion). I will assume the existence of a specialized working memory system for language and only 
briefly review the major motivating evidence in favor of it. Such evidence comes from independence of measures of verbal working memory capacity and sentence comprehension when meta-linguistic task requirements are factored out (see Caplan \& Waters, 1999, 2013 for reviews) and the existence of patients that have severely impaired verbal working memory capacity yet near normal sentence comprehension (Waters et al., 1991). In addition, modern models of working memory for sentence processing motivated by psycholinguistic research consist of a content-addressable memory retrieval system operating over syntactic representations in long-term memory (McElree et al., 2003; Lewis \& Vasishth, 2005; Caplan \& Waters, 2013) (Fig. 3). Given that the representations and retrieval operations in such a system are syntactic, these systems are by definition domain-specific, yet rely on domain-general organizational principles (Anderson \& Lebiere, 2014; Anderson et al., 2004). These models have been successful in accounting for behavioral data from both patients and healthy subjects in psycholinguistic experiments (see Lewis et al. 2006 and Caplan \& Waters, 2013 for reviews), have explained the existence of grammatical illusions, such as agreement attraction (Badecker \& Kuminiak, 2007; Wagers et al., 2009), and provide insight into the nature of language deficits in agrammatic Broca's aphasia (Matchin \& Rogalsky, in review). Thus, a language-specific working memory system with a basic character as described in Lewis et al. (2006) is well-motivated across fields.
A key component of this retrieval approach to working memory concerns the syntactic representations that are stored in long-term memory. Most computational models of sentence comprehension include stored structural representations of some form (Vosse \& Kempen, 2000; Lewis \& Vasishth, 2005; Demberg et al., 2013; Hale, 2001), whether conceived of as lexicalized syntactic trees or phrase structure rules. The upshot is that syntactic representations are valid targets of topdown activation in the same sense that phonological units or visual objects are, creating a parallelism among these domains with respect to working memory (Momma \& Phillips, 2018).

I posit that the pTri and the posterior superior temporal sulcus (pSTS) jointly underlie this syntactic working memory system. In neuroimaging studies, the pTri and the pSTS have a tightly coupled response profile (Pallier et al., 2011; Matchin et al., 2017; Wilson et al., 2010; Nelson et al., 2017; see Meyer \& Friederici, 2016 for a meta-analysis of embedding and noncanonical word order revealing these two regions), and degeneration of these regions is associated with syntactic deficits (Wilson et al., 2012). Given that posterior temporal lesions are strongly associated with sentence comprehension deficits (Dronkers et al., 2004; Pillay et al., 2017) and deficits in judging the structural well-formedness of sentences (Wilson \& Saygin, 2004), the pSTS likely underlies the storage of syntactic representations in long-term memory, whereas the pTri underlies the top-down retrieval of these representations. During sentence comprehension, overt speech, text or manual

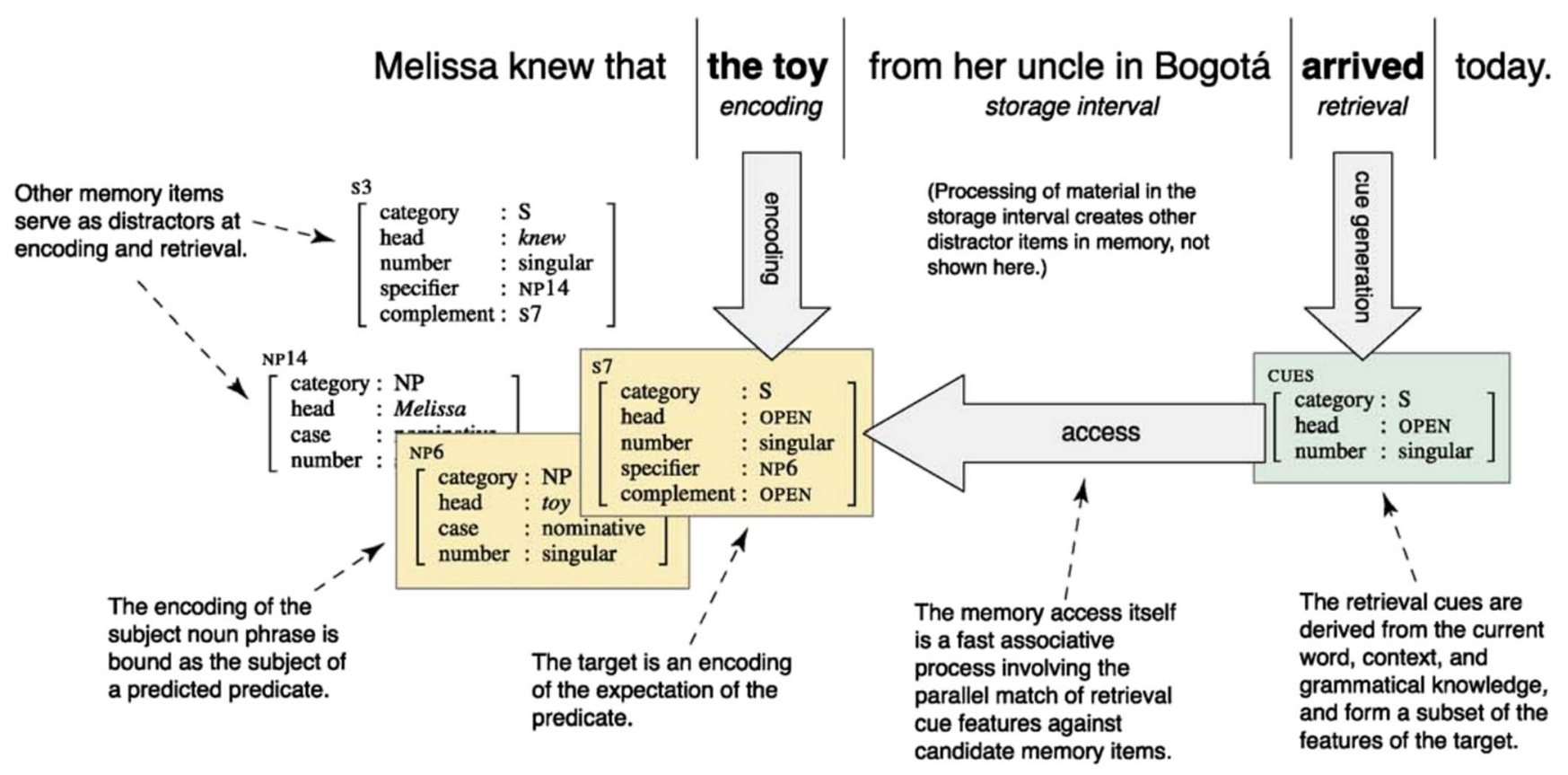

$\overline{T R E N D S ~ i n ~ C o g n i t i v e ~ S c i e n c e s ~}$

Fig. 3 Content-addressable retrieval model of syntactic working memory (adapted with permission from Lewis et al., 2006). Linguistic representations consist of "chunks" containing syntactic features that serve as targets for a parallel content-addressable memory system that makes use of the same set of syntactic features. 
sign activates syntactic representations in the pSTS, allowing for basic sentence comprehension. However, when information previously encountered must be retrieved (e.g., recovering the antecedent of an anaphor, subject-verb agreement), top-down memory retrieval access is performed via the pTri to re-activate the appropriate syntactic representation in the pSTS. Top-down memory access may also be useful for making predictions of upcoming material, which would be useful in sentence comprehension during difficult processing conditions. In support of this, patients with LIFG lesions including the pTri and surrounding regions (but excluding damage to temporal areas) showed a delayed ERP signature associated with subject-verb agreement violations (Jakuszeit et al., 2013), suggesting that they were unable to take advantage of predictive preactivation of the appropriate syntactic representation.

The pTri is localized in-between the pOrb and pOper, indicating that a common top-down memory retrieval function may be shared among these regions. This function can be thought of as an attentional mechanism, producing sustained activation for representations stored in long-term memory, as has been proposed for spatial vision (Awh \& Jonides, 2001). The pOrb has been strongly linked to semantic retrieval (see Badre \& Wagner, 2007 for a review), which can be thought of as a semantic attentional system, producing sustained activity of semantic representations in the temporal and parietal lobe. I review the relation between the pOper and verbal working memory, suggesting a similar role for this region in topdown activation of phonological representations. The pOper is strongly linked to phonological processing, particularly speech production (Hickok \& Poeppel, 2007; Rauschecker \& Scott, 2009; Tourville \& Guenther, 2011). Evidence for the involvement of the pOper in phonological processing comes from neuroimaging studies showing activation for subvocal speech production (Matchin et al., 2014; Okada et al., 2017), particularly for complex speech sequences (Bohland \& Guenther, 2006). In addition, this area shows sustained activity when verbal material is rehearsed (Hickok et al., 2003; Buchsbaum et al., 2011), supporting a role for this region in phonological working memory. I suggest that this role in working memory derives from a top-down attention mechanism applied to phonological representations stored in secondary auditory cortex. Experiments of subvocal speech production in magnetoencephalography (MEG) and fMRI have shown activity in secondary auditory cortex, suggesting that phonological representations are activated in absence of direct sensory input during production (Tian \& Poeppel, 2010; Okada et al., 2017). In addition, an ECoG experiment by Flinker et al. (2015) showed that activity in Broca's area (on the border of pTri and pOper) modulated activity in the superior temporal lobe during speech a production task. Interestingly, Broca's area did not activate directly during speech articulation, suggesting a role for higher-level planning of speech, which is consistent with a role in top-down activation of phonological representations in sensory cortex. This mechanism would be useful for both speech production (prediction of sensory targets for speech production) and sentence comprehension, i.e., verbal working memory (e.g., retrieval of previously heard speech for syntactic reanalysis, Kush et al., 2015). Therefore, there is good evidence to indicate a parallelism of top-down retrieval or attention mechanisms for semantic (pOrb), syntactic (roughly pTri), and phonological (pOper) representations and that all three are involved in sentence comprehension, although their contributions are distinct. While the computations are in parallel in each region, there may be a hierarchical organization among them, given that the relevant phonological representations may operate at a lower level of representation than syntax, and syntax a lower level than semantics.

I have argued here that the LIFG is partitioned into three distinct systems operating over phonological, syntactic, and semantic information. This proposal bears some similarity to Hagoort (2005, 2013), who suggested the same tripartite division with respect to basic compositional operations called unification (Jackendoff, 2002). I believe that the present proposal better accounts for the wide range of empirical data across fields, particularly because damage to this region does not impair basic sentence processing, as would be expected for a brain area involved in unification. In addition, this account connects well with the conclusions and explicit models from other fields, notably psycholinguistics of sentence processing (Lewis et al., 2006; Caplan \& Waters, 2013) as well as motor control (Hickok et al., 2011; Tourville \& Guenther, 2011; Tian \& Poeppel, 2010), facilitating inferences and scientific development across fields. Regardless, both the Hagoort $(2005,2013)$ and the present account must consider why this tripartite organization exists; that is, why is there such a parallelism of function across three adjacent subregions of the LIFG? I address this question in the following section, suggesting that the pTri acquired its specificity for syntactic representations through a process of neuronal retuning.

\section{Neuronal retuning}

The term neuronal retuning is used here to encompass two related notions: (i) phylogenetic or evolutionary repurposing (Jacob, 1977), called exaptation (Gould \& Vrba, 1982), and (ii) ontogenetic or developmental repurposing, called neuronal recycling (Dehaene \& Cohen, 2007). Assume that semantic and phonological retrieval are functions that are precursors to human language. With respect to the pTri, the function of top-down memory retrieval was "borrowed" from neighboring neural territory, the pOper (phonological) and the pOrb (semantic), and applied to a novel domain, namely syntactic features (as in Lewis et al., 2006). This borrowing could have 
occurred during evolution (exaptation), that is, a mutation occurred that exapted the retrieval function in pOper/pOrb to syntax, meaning that the syntactic specificity of the pTri is innate. The borrowing also could have occurred during development (neuronal recycling), meaning that this specificity is not innate but emerges while the organism is developing. This could have possibly occurred via the connections between the pTri and the pSTS, a region that appears central to language. There are reasons to suspect that the pTri is a case of neuronal recycling rather than exaptation. I review the example of the visual word form area (VWFA), a brain region that is specialized for reading (Dehaene \& Cohen, 2011). This region is an illustrative case of neuronal recycling given the very evolutionarily recent emergence of reading/writing in humans $\sim 5,000$ years ago (Schmandt-Besserat, 1996). I then outline a neuronal recycling proposal with respect to the $\mathrm{pTri}$ and the empirical motivation underlying it.

\section{The VWFA: a product of neuronal recycling}

The VWFA demonstrates unequivocally that a remarkable degree of functional specificity to brain areas can emerge from the environmental experience of an organism. The importance of experience does not imply that the function of cortical areas is equipotential and driven purely by environmental inputs; the example of the VWFA clearly illustrates that the computational role and functional specificity of brain areas arises as an interaction of experience and innate functional properties.

Dehaene and colleagues have revealed the orthographyspecificity of the VWFA through a series of neuroimaging experiments (Dehaene et al., 2010; Cohen et al., 2000). Such experiments have found that the VWFA responds selectively to orthographic forms over visually similar nonorthographic stimuli, and the extent of response-specificity correlates with literacy, or extent of developmental exposure to written language (Fig. 4), suggesting that this response-specificity is driven by developmental exposure to reading and writing. The VWFA occupies the end of a posterior-anterior gradient in the ventral stream along which the specificity of responses increases from equal preference to letters and nonletter control stimuli to selective preference for word-like forms (Vinckier et al., 2007).

The location and organization of hierarchical responses of the VWFA in the left hemisphere in the lateral occipitotemporal sulcus is highly reproducible across subjects, languages, and experimental paradigms (Dehaene \& Cohen,

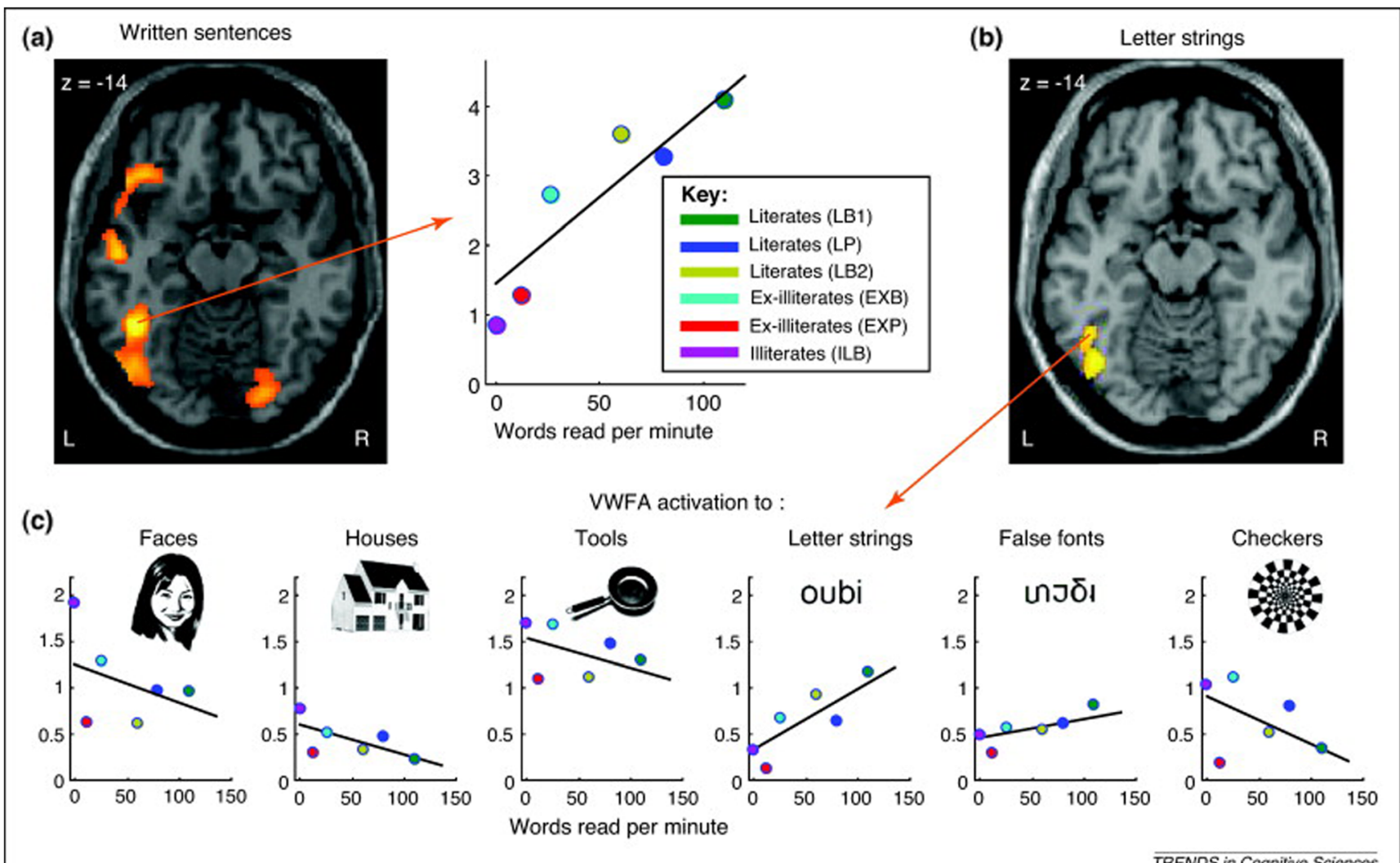

Fig. 4 The VWFA and orthography activation-selectivity correlation with literacy (adapted with permission from Dehaene \& Cohen, 2011). The VWFA activates as a function of exposure to written stimuli. (a) The magnitude of activation in the VWFA to written sentences increases as a function of literacy. (b) The VWFA activates to letter strings and (c) does not show increasing activation as a function of literacy for nonorthographic stimuli but does show this pattern for orthography 
2011), raising questions as to how this culturally derived functional region finds its neuronal "niche"-why is the VWFA consistently localized across individuals to this cortical location? Dehaene and Cohen (2007) postulate that the VWFA's consistent anatomical localization is due to three factors: (i) hierarchical activation gradient consistent with hierarchical coding in perceptual systems generally and the ventral occipitaltemporal cortex specifically, (ii) position in foveal cortex for fine resolution of orthographic features, (iii) and left hemisphere lateralization due to interaction with language, assumed to be left dominant. Thus, they highlight the importance of innate constraints on culturally derived functional maps through their framework of neuronal recycling: functional specificity does not appear randomly in the cortex but "recycles" a portion of the computationally appropriate region.

More generally, there appear to be two core factors underlying response specificity in regions of the cortex: innate (genetic) computational constraints and environmental inputs. The first principle constrains the type of computations the region performs. The second principle posits that a region initially nonspecific to a particular representational domain may become functionally specialized for that domain through developmental influences. Repeated exposure and cultural pressure to read early in life shapes the responseselectivity of the VWFA for orthography in connection with the rest of the language network. In turn, innate constraints on form processing in the brain constrain the development of human writing systems, limiting the range of systems to small sets of learnable symbol shapes that can be processed by these brain areas (Dehaene, 2009).

Instances of neuronal recycling have been found throughout the cortex. For instance, in the FFA, some researchers have reported car-specific responses for automobile collectors and bird-specific responses for bird watchers (Gauthier et al., 2000). A recent study (Andics et al., 2014) found that human voice-specific regions in the middle and posterior superior temporal sulcus (STS), referred to as "voice areas" (Belin et al., 2000), preferred dog vocalizations to nonvocal controls, suggesting that these regions are tuned to relevant sounds through experience. These findings are consistent with many examples of response tuning in single cortical neurons in nonhuman animals in sensory cortex, such as auditory, visual, and somatosensory areas (Bakin and Weinberger, 1990; Frégnac et al., 1988; Calford \& Tweedale, 1988). Given instances of environmental specialization throughout the brain, there could very well be retuning for language in Broca's area, because sentence processing may rely heavily on the phonological (oPper) and semantic (pOrb) resources that reside generally in the LIFG.

\section{The case for a neuronal recycling account of language-specificity in pTri}

The orthography-selectivity of the VWFA must be a product of neuronal recycling because of the recent emergence and cultural variation of writing systems. Conversely, the sentence-selectivity of the pTri might be; there certainly is no hard conceptual constraint as in the case of the VWFA. Despite this, developmental neuroimaging data provide some evidence in favor of a neuronal recycling account. Neuroimaging studies of speech perception in infants show activation in the classic perisylvian language areas, including posterior temporal and inferior frontal brain regions (see Dehaene-Lambertz 2017 for a review). These results suggest that both regions are involved in language early in development. In addition, anatomical studies comparing humans to nonhuman primates have revealed human-unique morphology of this system. Leroy et al. (2015) showed a unique leftright asymmetry of the superior temporal sulcus in humans compared with nonhuman primates. In addition, diffusion tensor imaging (DTI) studies have shown greatly expanded white matter tracks connecting the posterior temporal lobe to the IFG, namely, the arcuate fasciculus (AF) (Rilling et al., 2008). These data suggest that a pSTS-pTri circuit may underlie the core of human language in the brain. However, it appears that while the unique morphology of the STS is present in 3-month-old infants (Glasel et al., 2011), the AF is not mature until later in development. DTI studies comparing adults to infants have shown that the AF is relatively immature in children compared to other pathways (Dubois et al., 2006, 2008). Additionally, one study showed that there are white matter pathways connecting the posterior temporal lobe to the premotor cortex in both infants and adults but that the AF pathway connecting the posterior temporal lobe to Broca's area proper was not present in infants (Perani et al., 2011). This suggests that the sentence-specificity of the pTri may track with the development of white matter connecting it with the pSTS. In addition, the relative strength of languagerelated white matter tracks varies across languages: German speakers have a stronger AF, whereas English speakers have stronger white matter tracks running along the temporal lobe (Goucha et al., 2015). The freer word order of German and presence of much richer overt morphology may place greater demand on syntactic working memory resources greater than in English. This suggests that the functional-anatomical properties of the syntactic working memory system may depend on developmental experience.

These data are roughly consistent with the neuronal recycling account; however, additional data and specific details regarding how exactly this development occurs are needed in future research to better support this hypothesis. One way to test the neuronal recycling hypothesis would be to correlate behavioral measures of facility in sentence 
comprehension with extent of sentence-specificity in pTri and/ or the strength of white matter tracks connecting this region to the temporal lobe. Significant positive correlation among these measures would indicate that specificity tracks with experience, as shown for the VWFA (Dehaene et al., 2010). Additionally, the degree of sentence-specificity should change through the course of development; indices of degree of sentence-specificity could be developed and tested.

Fedorenko (2014) also has proposed a neuronal recycling account of sentence-specificity in Broca's area. However, Fedorenko suggests that the spatial segregation of the domain-specific and domain-general activations found in Broca's area implies that "the computations they perform are likely to be different given their different response profiles" [pg. 5, emphasis mine]. This is a misreading of neuronal recycling; by definition, neuronal recycling posits a relation between the precursor and novel functions (Dehaene \& Cohen, 2007). This requires specifying this shared function or property. In the case of the VWFA, the same computation (hierarchical perception) underlies both the old and new capacities, with the difference being the nature of the representations operated over (words vs. faces or objects). Another striking example of this is the study of neural plasticity in deaf cats by Lomber and colleagues (Lomber et al., 2010). This study showed that deaf cats show functional enhancement for motion detection and object localization that was eliminated when particular areas of auditory cortex were temporarily deactivated. This plasticity was functionally constrained; the relevant auditory areas that provided perceptual enhancement for visual motion detection and localization are normally involved in auditory motion detection and localization in hearing cats. In understanding the plasticity of these regions in deaf cats, it is essential to understand the function that this region performs in hearing cats and its relation to the new function in the deaf cats. In this light, the computations of adjacent subregions of Broca's area (pOper, pTri, pOrb) are quite possibly the same despite their differences in input-selectivity. This is why I argue that top-down memory retrieval operations, specialized for syntactic representations, are the mechanisms that underlie sentence-specificity in pTri, rather than an independent core linguistic function.

Whether the sentence-specificity in pTri occurs due to neuronal recycling or exaptation, taking the neuronal retuning idea seriously leaves many important things unknown about the relation between language and brain, particularly how hierarchical syntax structure building is realized in neural networks. That is, if the neuronal retuning view is correct, and the pTri is the locus of working memory resources rather than structure building operations, new ideas must by explored about the neural localization of syntax. Whether this specific retuning hypothesis is correct, the argument against syntax in Broca's area is supported by the robust data concerning intact syntactic abilities in patients with damage to this region. This raises the natural (and important) question: what brain area does underlie core linguistic computations? There are three possible answers to this question.

First, several previous authors have argued that the anterior temporal lobe (ATL) may be involved in syntax because of its reliable increased response to linguistic structure (Mazoyer et al., 1993; Humphries et al., 2005; Rogalsky \& Hickok, 2009; Brennan et al., 2012). However, damage to this region does not impair sentence comprehension and syntactic abilities but rather semantic ones (Hodges et al., 1992; Wilson et al., 2014; Mesulam et al., 2015; Schwartz et al., 2009; Jefferies \& Lambon Ralph, 2006). It may be the case that this region's function is involved in conceptual-semantic combination rather than basic syntax, as is consistent with MEG studies showing effects of combinatory semantics in the ATL (Bemis \& Pylkkanen, 2011; Pylkkanen et al., 2014) but not certain syntactic combinations (Del Prato \& Pylkkänen, 2014).

Second, the PTL, particularly the pSTS, also is a candidate for the cortical locus of syntactic operations. As discussed, damage to this region is highly associated with sentencelevel deficits (Dronkers et al., 2004; Wilson et al., 2010; Pillay et al., 2017; Wilson \& Saygin, 2004). The anatomical studies reviewed above indicate that both the morphology of the STS (Leroy et al., 2015) and the connectivity of frontal regions to the posterior temporal lobe (Rilling et al., 2008) are human-specific; given that language is a human-specific trait, these data point to the pSTS for core linguistic computations. However, by contrast with the ATL, the pSTS does not always activate for linguistic structure (Humphries et al., 2006; Rogalsky \& Hickok, 2009; see Matchin et al., 2017 for discussion), raising questions about this interpretation; pinning down the possible role for syntactic operations in the pSTS should be an important goal of future research in this area.

Finally, there may simply not be a "syntax area" in the brain. Syntactic computations might correspond to some other level of neural granularity (see Poeppel \& Embick; 2005; Embick \& Poeppel, 2015 for in-depth discussion of this issue). For example, some authors have proposed that cortical oscillations might underlie syntactic computations (Ding et al., 2016; Boeckx \& Benítez-Burraco, 2014). One can certainly imagine other alternative neural codes for core linguistic computations. These should be explored as candidates for basic syntactic mechanisms, particularly if the evidence does not support a role for syntax in the chief candidates of the pTri, the ATL and the pSTS.

\section{Conclusions}

Sentence-selective activation in the pars triangularis of Broca's area does not indicate core linguistic computations 
but rather reflects retuning of phonological and/or semantic working memory resources in the pars opercularis and the pars orbitalis for syntactic representations. This proposal connects research among disparate fields (psycholinguistics, aphasia, neuroimaging, motor control) and provides avenues for further exploration of how language is organized in the brain. The proposal that working memory resources specialized for language emerge in development is consonant with the goals of the Minimalist Program (Chomsky, 1995, 2005): namely, to reduce innate language-specific machinery to a minimum, and to illustrate how the adult capacity for language emerges from the interaction of this minimal language-specific machinery and domain-general mechanisms. If the present proposal is correct, a major remaining question is how syntactic representations emerge in the posterior temporal lobe, and how they are combined to create novel expressions. Sentence-selective activations in neuroimaging studies are certainly helpful in understanding the nature of the brain circuits involved in core aspects of language, but restricting the search to these areas may preclude the investigation into brain areas that are involved in syntactic operations but may not to show a sentence-selective response. Conversely, understanding the general computational properties of neural circuits in disparate cognitive domains may prove useful for identifying the circuits relevant for language.

Author note The author thanks Gregory Hickok for reading and commenting on several previous versions of this manuscript and two reviewers for their feedback. This work was supported by NIH grant DC03681 awarded to Gregory Hickok while the author was a graduate student at UC Irvine.

\section{References}

Amunts, K., Lenzen, M., Friederici, A. D., Schleicher, A., Morosan, P., Palomero-Gallagher, N., \& Zilles, K. (2010). Broca's region: Novel organizational principles and multiple receptor mapping. PLoS Biology, 8(9), e1000489.

Anderson, J. R., Bothell, D., Byrne, M. D., Douglass, S., Lebiere, C., \& Qin, Y. (2004). An integrated theory of the mind. Psychological Review, 111(4), 1036.

Anderson, J. R., \& Lebiere, C. J. (2014). The atomic components of thought. Abingdon:Psychology Press.

Andics, A., Gácsi, M., Faragó, T., Kis, A., \& Miklósi, Á. (2014). Voicesensitive regions in the dog and human brain are revealed by comparative fMRI. Current Biology, 24(5), 574-578.

Awh, E., \& Jonides, J. (2001). Overlapping mechanisms of attention and spatial working memory. Trends in Cognitive Sciences, 5(3), 119126

Baddeley, A. (2003). Working memory: Looking back and looking forward. Nature Reviews. Neuroscience, 4(10), 829.

Baddeley, A. D., \& Hitch, G. (1974). Working memory. Psychology of Learning and Motivation, 8, 47-89.
Badecker, W., \& Kuminiak, F. (2007). Morphology, agreement and working memory retrieval in sentence production: Evidence from gender and case in Slovak. Journal of Memory and Language, 56(1), 65-85.

Badre, D., \& Wagner, A. D. (2007). Left ventrolateral prefrontal cortex and the cognitive control of memory. Neuropsychologia, 45(13), 2883-2901.

Bakin, J. S., \& Weinberger, N. M. (1990). Classical conditioning induces CS-specific receptive field plasticity in the auditory cortex of the guinea pig. Brain Research, 536(1), 271-286.

Barton, J. J. (2008). Structure and function in acquired prosopagnosia: Lessons from a series of 10 patients with brain damage. Journal of Neuropsychology, 2(1), 197-225.

Bedny, M., Pascual-Leone, A., Dodell-Feder, D., Fedorenko, E., \& Saxe, R. (2011). Language processing in the occipital cortex of congenitally blind adults. Proceedings of the National Academy of Sciences, 108(11), 4429-4434.

Belin, P., Zatorre, R. J., Lafaille, P., Ahad, P., \& Pike, B. (2000). Voiceselective areas in human auditory cortex. Nature, 403(6767), 309312.

Bemis, D. K., \& Pylkkänen, L. (2011). Simple composition: A magnetoencephalography investigation into the comprehension of minimal linguistic phrases. The Journal of Neuroscience, 31(8), 2801-2814.

Boeckx, C. A., \& Benítez-Burraco, A. (2014). The shape of the human language-ready brain. Frontiers in Psychology, 5, 282.

Bohland, J. W., \& Guenther, F. H. (2006). An fMRI investigation of syllable sequence production. NeuroImage, 32(2), 821-841.

Bolhuis, J. J., Tattersall, I., Chomsky, N., \& Berwick, R. C. (2014). How could language have evolved? PLoS Biology, 12(8), e1001934.

Bornkessel, I., Zysset, S., Friederici, A. D., von Cramon, D. Y., \& Schlesewsky, M. (2005). Who did what to whom? The neural basis of argument hierarchies during language comprehension. NeuroImage, 26(1), 221-233.

Bornkessel-Schlesewsky, I., \& Schlesewsky, M. (2013). Reconciling time, space and function: A new dorsal-ventral stream model of sentence comprehension. Brain and Language, 125(1), 60-76.

Braver, T. S., Cohen, J. D., Nystrom, L. E., Jonides, J., Smith, E. E., \& Noll, D. C. (1997). A parametric study of prefrontal cortex involvement in human working memory. NeuroImage, 5(1), 49-62.

Brennan, J., Nir, Y., Hasson, U., Malach, R., Heeger, D. J., \& Pylkkänen, L. (2012). Syntactic structure building in the anterior temporal lobe during natural story listening. Brain and Language, 120(2), 163173.

Buchsbaum, B. R., Baldo, J., Okada, K., Berman, K. F., Dronkers, N., D'Esposito, M., \& Hickok, G. (2011). Conduction aphasia, sensorymotor integration, and phonological short-term memory-an aggregate analysis of lesion and fMRI data. Brain and Language, 119(3), 119-128.

Calford, M. B., \& Tweedale, R. (1988). Immediate and chronic changes in responses of somatosensory cortex in adult flying-fox after digit amputation. Nature, 332(6163), 446-448.

Caplan, D., \& Waters, G. (2013). Memory mechanisms supporting syntactic comprehension. Psychonomic Bulletin \& Review, 20(2), 243268.

Caplan, D., \& Waters, G. S. (1999). Verbal working memory and sentence comprehension. Behavioral and Brain Sciences, 22(01), 7794.

Caramazza, A., \& Zurif, E. B. (1976). Dissociation of algorithmic and heuristic processes in language comprehension: Evidence from aphasia. Brain and Language, 3(4), 572-582.

Chomsky, N. (1959). A review of BF Skinner's Verbal Behavior. Language, 35(1), 26-58.

Chomsky, N. (1965). Aspects of the Theory of Syntax. Cambridge, MA: MIT press.

Chomsky, N. (1995). The Minimalist Program. Cambridge, MA: MIT press. 
Chomsky, N. (2005). Three factors in language design. Linguistic Inquiry, $36(1), 1-22$.

Cohen, L., Dehaene, S., Naccache, L., Lehéricy, S., Dehaene-Lambertz, G., Hénaff, M. A., \& Michel, F. (2000). The visual word form area Spatial and temporal characterization of an initial stage of reading in normal subjects and posterior split-brain patients. Brain, 123(2), 291-307.

Curtis, C. E., \& D'Esposito, M. (2003). Persistent activity in the prefrontal cortex during working memory. Trends in Cognitive Sciences, 7(9), 415-423.

Davis, C., Kleinman, J. T., Newhart, M., Gingis, L., Pawlak, M., \& Hillis, A. E. (2008). Speech and language functions that require a functioning Broca's area. Brain and Language, 105(1), 50-58.

Dehaene, S. (2009). Reading in the brain: The new science of how we read. New York: Penguin.

Dehaene, S., \& Cohen, L. (2007). Cultural recycling of cortical maps. Neuron, 56(2), 384-398.

Dehaene, S., \& Cohen, L. (2011). The unique role of the visual word form area in reading. Trends in Cognitive Sciences, 15(6), 254-262.

Dehaene, S., Pegado, F., Braga, L. W., Ventura, P., Nunes Filho, G., Jobert, A., .. \& Cohen, L. (2010). How learning to read changes the cortical networks for vision and language. Science, 330(6009), $1359-1364$.

Dehaene-Lambertz, G. (2017). The human infant brain: A neural architecture able to learn language. Psychonomic Bulletin \& Review, 24(1), 48-55.

Del Prato, P., \& Pylkkänen, L. (2014). MEG evidence for conceptual combination but not numeral quantification in the left anterior temporal lobe during language production. Frontiers in Psychology, 5, 524.

Demberg, V., Keller, F., \& Koller, A. (2013). Incremental, predictive parsing with psycholinguistically motivated tree-adjoining grammar. Computational Linguistics, 39(4), 1025-1066.

Ding, N., Melloni, L., Zhang, H., Tian, X., \& Poeppel, D. (2016). Cortical tracking of hierarchical linguistic structures in connected speech. Nature Neuroscience, 19(1), 158-164.

Dronkers, N. F., Wilkins, D. P., Van Valin, R. D., Redfern, B. B., \& Jaeger, J. J. (2004). Lesion analysis of the brain areas involved in language comprehension. Cognition, 92(1), 145-177.

Dubois, J., Dehaene-Lambertz, G., Perrin, M., Mangin, J. F., Cointepas, Y., Duchesnay, E., ... \& Hertz-Pannier, L. (2008). Asynchrony of the early maturation of white matter bundles in healthy infants: Quantitative landmarks revealed noninvasively by diffusion tensor imaging. Human Brain Mapping, 29(1), 14-27.

Dubois, J., Hertz-Pannier, L., Dehaene-Lambertz, G., Cointepas, Y., \& Le Bihan, D. (2006). Assessment of the early organization and maturation of infants' cerebral white matter fiber bundles: A feasibility study using quantitative diffusion tensor imaging and tractography. NeuroImage, 30(4), 1121-1132.

Embick, D., Marantz, A., Miyashita, Y., O'Neil, W., \& Sakai, K. L. (2000). A syntactic specialization for Broca's area. Proceedings of the National Academy of Sciences, 97(11), 6150-6154.

Embick, D., \& Poeppel, D. (2015). Towards a computational (ist) neurobiology of language: Correlational, integrated and explanatory neurolinguistics. Language, Cognition and Neuroscience, 30(4), 357-366.

Fedorenko, E. (2014). The role of domain-general cognitive control in language comprehension. Frontiers in Psychology, 5:335.

Fedorenko, E., Duncan, J., \& Kanwisher, N. (2012). Language-selective and domain-general regions lie side by side within Broca's area. Current Biology, 22(21), 2059-2062.

Fedorenko, E., Gibson, E., \& Rohde, D. (2007). The nature of working memory in linguistic, arithmetic and spatial integration processes. Journal of Memory and Language, 56(2), 24.

Fedorenko, E., \& Thompson-Schill, S. L. (2014). Reworking the language network. Trends in Cognitive Sciences, 18(3), 120-126.
Fiebach, C. J., Schlesewsky, M., Lohmann, G., Von Cramon, D. Y., \& Friederici, A. D. (2005). Revisiting the role of Broca's area in sentence processing: Syntactic integration versus syntactic working memory. Human Brain Mapping, 24(2), 79-91.

Flinker, A., Korzeniewska, A., Shestyuk, A. Y., Franaszczuk, P. J., Dronkers, N. F., Knight, R. T., \& Crone, N. E. (2015). Redefining the role of Broca's area in speech. Proceedings of the National Academy of Sciences, 112(9), 2871-2875.

Frank, R. (2002). Phrase structure composition and syntactic dependencies. Cambridge, MA: MIT Press.

Frégnac, Y., Shulz, D., Thorpe, S., \& Bienenstock, E. (1988). A cellular analogue of visual cortical plasticity. Nature, 333(6171), 367-370.

Friederici, A. D. (2011). The brain basis of language processing: From structure to function. Physiological Reviews, 91(4), 1357-1392.

Friederici, A. D., Bahlmann, J., Heim, S., Schubotz, R. I., \& Anwander, A. (2006). The brain differentiates human and non-human grammars: Functional localization and structural connectivity. Proceedings of the National Academy of Sciences of the United States of America, 103(7), 2458-2463.

Gauthier, I., Skudlarski, P., Gore, J. C., \& Anderson, A. W. (2000). Expertise for cars and birds recruits brain areas involved in face recognition. Nature Neuroscience, 3(2), 191-197.

Gibson, E. (1998). Linguistic complexity: Locality of syntactic dependencies. Cognition, 68(1), 1-76.

Glasel, H., Leroy, F., Dubois, J., Hertz-Pannier, L., Mangin, J. F., \& Dehaene-Lambertz, G. (2011). A robust cerebral asymmetry in the infant brain: The rightward superior temporal sulcus. NeuroImage, 58(3), 716-723.

Glaser, Y. G., Martin, R. C., Van Dyke, J. A., Hamilton, A. C., \& Tan, Y. (2013). Neural basis of semantic and syntactic interference in sentence comprehension. Brain and Language, 126(3), 314-326.

Goldberg, A. E. (1995). Constructions: A construction grammar approach to argument structure. Chicago: University of Chicago Press.

Goldman-Rakic, P. S. (1995). Cellular basis of working memory. Neuron, 14(3), 477-485.

Goucha, T., Anwander, A., \& Friederici, A. D. (2015). How language shapes the brain: Cross-linguistic differences in structural connectivity. Poster presented at 45th Annual Meeting of the Society for Neuroscience (SfN 2015), Chicago, IL, USA.

Gould, S. J., \& Vrba, E. S. (1982). Exaptation - a missing term in the science of form. Paleobiology, 8, 4-15.

Grodzinsky, Y. (2000). The neurology of syntax: Language use without Broca's area. Behavioral and Brain Sciences, 23(01), 1-21.

Grodzinsky, Y., \& Santi, A. (2008). The battle for Broca's region. Trends in Cognitive Sciences, 12(12), 474-480.

Hagoort, P. (2005). On Broca, brain, and binding: A new framework. Trends in Cognitive Sciences, 9(9), 416-423.

Hagoort, P. (2013). MUC (memory, unification, control) and beyond. Frontiers in Psychology, 4:416.

Hale, J. (2001). A probabilistic Earley parser as a psycholinguistic model. In Proceedings of the second meeting of the North American Chapter of the Association for Computational Linguistics on Language technologies(pp. 1-8). Association for Computational Linguistics.

Hauser, M. D., Chomsky, N., \& Fitch, W. T. (2002). The faculty of language: What is it, who has it, and how did it evolve? Science, 298(5598), 1569-1579.

Hickok, G., Buchsbaum, B., Humphries, C., \& Muftuler, T. (2003). Auditory-motor interaction revealed by fMRI: Speech, music, and working memory in area Spt. Journal of Cognitive Neuroscience, 15(5), 673-682.

Hickok, G., Houde, J., \& Rong, F. (2011). Sensorimotor integration in speech processing: Computational basis and neural organization. Neuron, 69(3), 407422. 
Hickok, G., \& Poeppel, D. (2007). The cortical organization of speech processing. Nature Reviews Neuroscience, 8(5), 393-402.

Hodges, J. R., Patterson, K., Oxbury, S., \& Funnell, E. (1992). Semantic dementia. Brain, 115(6), 1783-1806.

Humphries, C., Binder, J. R., Medler, D., \& Liebenthal, E. (2006). Syntactic and semantic modulation of neural activity during auditory sentence comprehension. Journal of Cognitive Neuroscience, 18(4), 665-679.

Humphries, C., Love, T., Swinney, D., \& Hickok, G. (2005). Response of anterior temporal cortex to syntactic and prosodic manipulations during sentence processing. Human Brain Mapping, 26(2), 128138.

Jackendoff, R. (2002). Foundations of language: Brain, meaning, grammar, evolution. Oxford: Oxford University Press.

Jacob, F. (1977). Evolution and tinkering. Science, 196, 1161-1166.

Jakuszeit, M., Kotz, S. A., \& Hasting, A. S. (2013). Generating predictions: Lesion evidence on the role of left inferior frontal cortex in rapid syntactic analysis. Cortex, 49(10), 2861-2874.

Jefferies, E., \& Lambon Ralph, M. A. (2006). Semantic impairment in stroke aphasia versus semantic dementia: A case-series comparison. Brain, 129(8), 2132-2147.

Just, M. A., \& Carpenter, P. A. (1992). A capacity theory of comprehension: Individual differences in working memory. Psychological Review, 99(1), 122

Kaan, E., \& Swaab, T. Y. (2002). The brain circuitry of syntactic comprehension. Trends in Cognitive Sciences, 6(8), 350-356.

Kanwisher, N., McDermott, J., \& Chun, M. M. (1997). The fusiform face area: A module in human extrastriate cortex specialized for face perception. The Journal of Neuroscience, 17(11), 4302-4311.

Kush, D., Johns, C. L., \& Van Dyke, J. A. (2015). Identifying the role of phonology in sentence-level reading. Journal of Memory and Language, 79, 18-29.

Leroy, F., Cai, Q., Bogart, S. L., Dubois, J., Coulon, O., Monzalvo, K., ... $\&$ Lin, C. P. (2015). New human-specific brain landmark: The depth asymmetry of superior temporal sulcus. Proceedings of the National Academy of Sciences, 112(4), 1208-1213.

Lewis, R. L., \& Vasishth, S. (2005). An activation-based model of sentence processing as skilled memory retrieval. Cognitive Science, 29(3), 375-419.

Lewis, R. L., Vasishth, S., \& Van Dyke, J. A. (2006). Computational principles of working memory in sentence comprehension. Trends in Cognitive Sciences, 10(10), 447-454.

Linebarger, M. C., Schwartz, M. F., \& Saffran, E. M. (1983). Sensitivity to grammatical structure in so-called agrammatic aphasics. Cognition, 13(3), 361-392.

Lomber, S. G., Meredith, M. A., \& Kral, A. (2010). Cross-modal plasticity in specific auditory cortices underlies visual compensations in the deaf. Nature Neuroscience, 13(11), 1421-1427.

Matchin, W., Hammerly, C., \& Lau, E. (2017). The role of the IFG and pSTS in syntactic prediction: Evidence from a parametric study of hierarchical structure in fMRI. Cortex, 88, 106-123.

Matchin, W. \& Rogalsky, C. (in review). Aphasia and syntax. In: The Handbook of Experimental Syntax. Sprouse, J. (editor). Oxford: Oxford University Press.

Matchin, W., Sprouse, J., \& Hickok, G. (2014). A structural distance effect for backward anaphora in Broca's area: An fMRI study. Brain and Language, 138, 1-11.

Mazoyer, B. M., Tzourio, N., Frak, V., Syrota, A., Murayama, N., Levrier, O., ... \& Mehler, J. (1993). The cortical representation of speech. Journal of Cognitive Neuroscience, 5(4), 467-479.

McCarthy, G., Puce, A., Gore, J. C., \& Allison, T. (1997). Face-specific processing in the human fusiform gyrus. Journal of Cognitive Neuroscience, 9(5), 605-610.

McElree, B., Foraker, S., \& Dyer, L. (2003). Memory structures that subserve sentence comprehension. Journal of Memory and Language, 48(1), 67-91.
Mesulam, M. M., Thompson, C. K., Weintraub, S., \& Rogalski, E. J. (2015). The Wernicke conundrum and the anatomy of language comprehension in primary progressive aphasia. Brain, 138(8), 2423-2437.

Meyer, L., \& Friederici, A. D. (2016). Neural systems underlying the processing of complex sentences. In G. Hickok, \& S. A. Small (Eds.), The Neurobiology of language. London: Elsevier.

Miller, E. K., Erickson, C. A., \& Desimone, R. (1996). Neural mechanisms of visual working memory in prefrontal cortex of the macaque. The Journal of Neuroscience, 16(16), 5154-5167.

Miller, G.A., Chomsky, N., 1963. Finitary models of language users. In: Luce, R.D., Bush, R.R., Galanter, E. (Eds.), Handbook of Mathematical Psychology, volume 2. New York: Wiley, pp. 419491.

Mohr, J. P., Pessin, M. S., Finkelstein, S., Funkenstein, H. H., Duncan, G. W., \& Davis, K. R. (1978). Broca aphasia Pathologic and clinical. Neurology, 28(4), 311-311.

Momma, S., \& Phillips, C. (2018). The Relationship Between Parsing and Generation. Annual Review of Linguistics, 4.

Moro, A., Tettamanti, M., Perani, D., Donati, C., Cappa, S. F., \& Fazio, F. (2001). Syntax and the brain: Disentangling grammar by selective anomalies. NeuroImage, 13(1), 110-118.

Murphy, B. L., Arnsten, A. F., Goldman-Rakic, P. S., \& Roth, R. H. (1996). Increased dopamine turnover in the prefrontal cortex impairs spatial working memory performance in rats and monkeys. Proceedings of the National Academy of Sciences, 93(3), 13251329.

Nelson, M. J., El Karoui, I., Giber, K., Yang, X., Cohen, L., Koopman, H., ... \& Dehaene, S. (2017). Neurophysiological dynamics of phrase-structure building during sentence processing. Proceedings of the National Academy of Sciences, 114(18).

Novick, J. M., Trueswell, J. C., \& Thompson-Schill, S. L. (2005). Cognitive control and parsing: Reexamining the role of Broca's area in sentence comprehension. Cognitive, Affective, \& Behavioral Neuroscience, 5(3), 263-281.

Okada, K., Matchin, W., \& Hickok, G. (2017). Neural evidence for predictive coding in auditory cortex during speech production. Psychonomic Bulletin \& Review, 1-8.

Pallier, C., Devauchelle, A. D., \& Dehaene, S. (2011). Cortical representation of the constituent structure of sentences. Proceedings of the National Academy of Sciences, 108(6), 2522-2527.

Perani, D., Saccuman, M. C., Scifo, P., Anwander, A., Spada, D., Baldoli, C., ... \& Friederici, A. D. (2011). Neural language networks at birth. Proceedings of the National Academy of Sciences, 108(38), 1605616061.

Pillay, S. B., Binder, J. R., Humphries, C., Gross, W. L., \& Book, D. S. (2017). Lesion localization of speech comprehension deficits in chronic aphasia. Neurology, 88(10), 970-975.

Pinker, S. (1995). The language instinct: The new science of language and mind (Vol. 7529). UK: Penguin.

Poeppel, D., \& Embick, D. (2005). Defining the relation between linguistics and neuroscience. In Cutler, A. (Ed.), Twenty-first century psycholinguistics: Four cornerstones (pp. 103-118). Psychology Press.

Pylkkänen, L., Bemis, D. K., \& Elorrieta, E. B. (2014). Building phrases in language production: An MEG study of simple composition. Cognition, 133(2), 371-384.

Rauschecker, J. P., \& Scott, S. K. (2009). Maps and streams in the auditory cortex: Nonhuman primates illuminate human speech processing. Nature Neuroscience, 12(6), 718-724.

Rilling, J. K., Glasser, M. F., Preuss, T. M., Ma, X., Zhao, T., Hu, X., \& Behrens, T. E. (2008). The evolution of the arcuate fasciculus revealed with comparative DTI. Nature Neuroscience, 11(4), 426.

Rogalsky, C., Almeida, D., Sprouse, J., \& Hickok, G. (2015). Sentence processing selectivity in Broca's area: Evident for structure but not syntactic movement. Language, Cognition and Neuroscience, 30(10), 1326-1338. 
Rogalsky, C., \& Hickok, G. (2009). Selective attention to semantic and syntactic features modulates sentence processing networks in anterior temporal cortex. Cerebral Cortex, 19(4), 786-796.

Rogalsky, C., \& Hickok, G. (2011). The role of Broca's area in sentence comprehension. Journal of Cognitive Neuroscience, 23(7), 16641680.

Rogalsky, C., Matchin, W., \& Hickok, G. (2008). Broca's area, sentence comprehension, and working memory: An fMRI study. Frontiers in Human Neuroscience, 214.

Saffran, J. R., Aslin, R. N., \& Newport, E. L. (1996). Statistical learning by 8-month-old infants. Science, 274 1926-1928.

Sahin, N. T., Pinker, S., Cash, S. S., Schomer, D., \& Halgren, E. (2009). Sequential processing of lexical, grammatical, and phonological information within Broca's area. Science, 326(5951), 445-449.

Saur, D., Schelter, B., Schnell, S., Kratochvil, D., Küpper, H., Kellmeyer, P., ... \& Mader, W. (2010). Combining functional and anatomical connectivity reveals brain networks for auditory language comprehension. NeuroImage, 49(4), 3187-3197.

Schmandt-Besserat, D. (1996). How writing came about. Austin: University of Texas Press.

Schwartz, M. F., Kimberg, D. Y., Walker, G. M., Faseyitan, O., Brecher, A., Dell, G. S., \& Coslett, H. B. (2009). Anterior temporal involvement in semantic word retrieval: Voxel-based lesion-symptom mapping evidence from aphasia. Brain, 132(12), 3411-3427.

Sprouse, J., \& Hornstein, N. (2016). Syntax and the cognitive neuroscience of syntactic structure building. In G. Hickok, \& S. A. Small (Eds.), The Neurobiology of Language. Elsevier, 167-174.

Stowe, L. A., Broere, C. A., Paans, A. M., Wijers, A. A., Mulder, G., Vaalburg, W., et al. (1998). Localizing components of a complex task: Sentence processing and working memory. NeuroReport, 9(13), 2995e2999

Stromswold, K., Caplan, D., Alpert, N., \& Rauch, S. (1996). Localization of syntactic comprehension by positron emission tomography. Brain and Language, 52(3), 452-473.

Thompson-Schill, S. L., D’Esposito, M., Aguirre, G. K., \& Farah, M. J. (1997). Role of left inferior prefrontal cortex in retrieval of semantic knowledge: A reevaluation. Proceedings of the National Academy of Sciences, 94(26), 14792-14797.

Thothathiri, M., Kimberg, D. Y., \& Schwartz, M. F. (2012). The neural basis of reversible sentence comprehension: Evidence from voxelbased lesion symptom mapping in aphasia. Journal of Cognitive Neuroscience, 24(1), 212-222.

Tian, X., \& Poeppel, D. (2010). Mental imagery of speech and movement implicates the dynamics of internal forward models. Frontiers in Psychology, 1166.
Tomasello, M. (2009). Constructing a language. Cambridge: Harvard University Press.

Tourville, J. A., \& Guenther, F. H. (2011). The DIVA model: A neural theory of speech acquisition and production. Language and cognitive processes, 26(7), 952-981.

Tremblay, P., \& Dick, A. S. (2016). Broca and Wernicke are dead, or moving past the classic model of language neurobiology. Brain and Language, 162, 60-71.

Vinckier, F., Dehaene, S., Jobert, A., Dubus, J. P., Sigman, M., \& Cohen, L. (2007). Hierarchical coding of letter strings in the ventral stream: Dissecting the inner organization of the visual word-form system. Neuron, 55(1), 143-156.

Vosse, T., \& Kempen, G. (2000). Syntactic structure assembly in human parsing: A computational model based on competitive inhibition and a lexicalist grammar. Cognition, 75(2), 105-143.

Wagers, M. W., Lau, E. F., \& Phillips, C. (2009). Agreement attraction in comprehension: Representations and processes. Journal of Memory and Language, 61(2), 206-237.

Waters, G., Caplan, D., \& Hildebrandt, N. (1991). On the structure of verbal short-term memory and its functional role in sentence comprehension: Evidence from neuropsychology. Cognitive Neuropsychology, 8(2), 81-126.

Wilson, S. M., DeMarco, A. T., Henry, M. L., Gesierich, B., Babiak, M., Mandelli, M. L., ... \& Gorno-Tempini, M. L. (2014). What role does the anterior temporal lobe play in sentence-level processing? Neural correlates of syntactic processing in semantic variant primary progressive aphasia. Journal of Cognitive Neuroscience, 26(5), 970985.

Wilson, S. M., Dronkers, N. F., Ogar, J. M., Jang, J., Growdon, M. E., Agosta, F., ... \& Gorno-Tempini, M. L. (2010). Neural correlates of syntactic processing in the nonfluent variant of primary progressive aphasia. Journal of Neuroscience, 30(50), 16845-16854.

Wilson, S. M., Galantucci, S., Tartaglia, M. C., \& Gorno-Tempini, M. L. (2012). The neural basis of syntactic deficits in primary progressive aphasia. Brain and Language, 122(3), 190-198.

Wilson, S. M., \& Saygın, A. P. (2004). Grammaticality judgment in aphasia: Deficits are not specific to syntactic structures, aphasic syndromes, or lesion sites. Journal of Cognitive Neuroscience, 16(2), 238-252.

Zaccarella, E., Meyer, L., Makuuchi, M., \& Friederici, A. D. (2017). Building by syntax: the neural basis of minimal linguistic structures. Cerebral Cortex, 27(1), 411-421.

Zahrt, J., Taylor, J. R., Mathew, R. G., \& Arnsten, A. F. (1997). Supranormal stimulation of D1 dopamine receptors in the rodent prefrontal cortex impairs spatial working memory performance. Journal of Neuroscience, 17(21), 8528-8535. 